\title{
Semaphorin signaling in morphogenesis: found in translation
}

\author{
Andrew D. Chisholm ${ }^{1}$ \\ Division of Biological Sciences, Section of Neurobiology, Section of Cell and Developmental Biology, University \\ of California at San Diego, La Jolla, California 92093, USA
}

Semaphorins play diverse roles in axon guidance and epithelial morphogenetic cell movements. In this issue of Genes \& Development, Nukazuka and colleagues (pp. 1025-1036) show that semaphorins regulate Caenorhabditis elegans male tail morphogenesis by stimulating the translation of specific messages, including the actin-depolymerizing enzyme cofilin.

Semaphorins form one of the major classes of intercellular signaling pathways in developmental biology. Initially defined by their repellent effects on axonal growth cones, semaphorins were found later to also attract growth cones (Polleux et al. 2000), and are now recognized as regulators of many nonneuronal developmental processes including morphogenesis of epithelial and endothelial tissues (Hinck 2004; Tran et al. 2007). Accompanying, and possibly explaining, this diversity of biological functions for semaphorins is a strikingly diverse array of signaling mechanisms. Most semaphorins exert their effects via a conserved family of transmembrane receptors, the plexins. Vertebrate-secreted semaphorins do not bind plexins directly, but instead usually bind obligate coreceptors called neuropilins, which then activate a semaphorin-neuropilin-plexin holoreceptor complex. The ability of neuropilins or plexins to couple to multiple coreceptors may explain the distinct readouts of semaphorin signaling (Tamagnone and Comoglio 2004): For example, Sema6D can promote or inhibit cell migration in cardiac morphogenesis depending on whether its receptor Plexin-A1 couples with two different receptor tyrosine kinases (Toyofuku et al. 2004). A major goal in analysis of semaphorin signaling is to understand how the different receptor complexes can have distinct (and sometimes opposing) biological effects.

How do semaphorin signaling pathways in morphogenetic movements compare with the more "canonical" pathways studied in growth cone repulsion? Semaphorininduced growth cone collapse involves disruption of the

[Keywords: C. elegans; cofilin; eIF2; epidermal morphogenesis; mRNA translation; semaphorin]

${ }^{1}$ Correspondence.

E-MAIL chisholm@ucsd.edu; FAX (858) 534-7773.

Article is online at http://www.genesdev.org/cgi/doi/10.1101/gad.1669308. local actin and tubulin cytoskeleton, coupled with inhibition of integrin-mediated adhesion. These cytoskeletal effects can be mediated by small GTPases, including Rac, Rho, and Ras (Tran et al. 2007). Additionally, it is now clear that axon guidance can involve local translation of cytoskeletal components and regulators within the growth cone or axon. Seminal work by the Holt laboratory (Campbell and Holt 2001) revealed that local translation was required for growth cones to be repelled by semaphorin $3 \mathrm{~A}$ in vitro. Not only is translation required for this response, but Sema3A can directly activate TOR kinase-mediated phosphorylation and inhibit the translational initiation repressor eIF4E-BP1. This likely results in general stimulation of translation by activation of eIF4E. Specificity arises from the localization of specific messages to growth cones, so that only axonally localized messages undergo stimulated translation.

Is translation stimulation used by semaphorins in their guises as cell adhesion or tissue remodeling factors? In this issue of Genes \& Development, Nukazuka et al. (2008) extend our understanding of the mechanism of semaphorin signaling in morphogenetic cell movements. Using a combination of genetic and biochemical analysis, they show that semaphorins stimulate translation in vivo to control an epidermal cell movement in Caenorhabditis elegans. Moreover, they find that semaphorins stimulate translation in responding cells via the initiation factor eIF $2 \alpha$, and that a major target of semaphorin-stimulated translation is the actin-severing enzyme ADF/cofilin.

\section{Semaphorin signaling in C. elegans epidermal morphogenesis}

Semaphorin signaling in C. elegans involves a relatively small number of players: three semaphorins, two plexins, and no neuropilins (Fig. 1A). Two related transmembrane semaphorins, SMP-1 and SMP-2, have partly redundant roles in signaling via the A-type plexin PLX-1 (Fujii et al. 2002; Dalpe et al. 2004). SMP-1 and SMP-2 are class I semaphorins, and are structurally similar to vertebrate class VI. The known functions of the SMP-1/2/ PLX-1 pathway are in morphogenesis of epidermal cells and epidermally derived sensilla in sexually dimorphic 
Figure 1. (A) Semaphorins and their receptors in C. elegans. Two class I semaphorins, SMP-1 and SMP-2, function partly redundantly in PLX-1 plexin signaling. The secreted class 2 semaphorin MAB-20 binds PLX-2 (Nakao et al. 2007); however, genetic data indicate PLX-2 must act in concert with other receptors. (B) Positioning of ray cells in the male tail epithelium. Schematic diagram of part of the lateral male tail, anterior to the left and dorsal up. Epidermal cells are in orange, and the ray cell group cells (two neurons and a structural cell) are blue. In the wild type, ray groups 1 and 2 form next to each other in the lateral epidermis; ray 1 's posterior position is dependent on attractive semaphorin signaling from the hook (green). Ray group 1 normally clusters at the junction between R1.p and R2.p (which later fuse). In animals lacking the semaphorin signal, the R1.p cell is smaller; as a consequence, the ray 1 cell group is separated from ray 2. A more anatomically correct view of ray formation is given at http://www.wormatlas.org. (C) Signaling from PLX-1 activates translation via eIF2 $\alpha$. PLX-1 reduces the level of phospho-eIF2 $\alpha$ by an unknown signaling pathway. GCN-1 and PEK-1 together increase phospho-eIF $2 \alpha$, either indirectly or directly.

structures in the male tail and hermaphrodite vulva (Liu et al. 2005). In contrast, loss of function in the secreted class 2 semaphorin MAB-20 has more drastic effects on neuronal development and epidermal morphogenesis (Roy et al. 2000). Perplexingly, deletion of the putative MAB-20 receptor, the divergent B-type plexin PLX-2, has only mild effects on development; MAB-20 signal reception appears to be complex and may involve cell-typedependent cross-talk with L1CAM (Wang et al. 2008) and the ephrin EFN-4 (Ikegami et al. 2004; Nakao et al. 2007).

Nukazuka et al. (2008) focus on perhaps the best-studied role of SMP/PLX-1 signaling, in positioning of the ray 1 sensillum in the male tail (schematically depicted in Fig. 1B). The C. elegans male tail is a complex sensory organ essential for male mating and contains nine bilaterally paired sensilla, known as rays, arrayed in stereotyped positions along the anteroposterior axis (Baird et al. 1991). Each ray sensillum develops from a single neuroepithelial precursor ("Rn cell") that divides to generate an epidermal cell, two neurons, and the structural cell of the sensillum. The final position of the sensillum within the epithelium is thought to be determined primarily by the adhesive contacts of its epidermal cell (Rn.p). Loss of function in plx-1 or in smp-1 smp-2 double mutants (hereafter, $s m p$ mutants) results in an abnormally shaped R1.p cell, leading to anterior mispositioning of ray 1 precursors and an anteriorly displaced ray 1 sensillum. Previous work suggested that ray 1 precursors (expressing PLX-1) are somehow attracted to SMP-expressing cells of a more posterior sensillum, the hook (Dalpe et al. 2004), although it has remained unclear if this involves direct contact between ray 1 cells and hook cells, or cleavage and release of the transmembrane SMPs and their local diffusion. Both the SMP/PLX1-dependent posterior attraction and SMP/PLX-1-independent anterior positioning may be active processes of migration, as both are dependent on Rac GTPase signal- ing depending on the genetic background. As ray 1 is normally positioned in $\sim 20 \%$ of $p l x-1$ - or smp-null mutants, a second pathway is thought to act in parallel to the SMP/PLX-1 pathway to allow ray 1 to reside in its normal posterior location.

\section{Genetic screens identify translational controls}

To find new components of the SMP/PLX-1 pathway Nukazuka et al. (2008) took a classical genetic approach; namely, to screen for second site suppressors of the $p l x-1$ ray 1 position defect. This screen netted a single allele of gcn-1, the C. elegans ortholog of the translational regulator GCN1. GCN1's function in translational control was elegantly dissected by genetic and biochemical analysis of amino acid starvation responses in yeast (Hinnebusch 2005). GCN1 regulates translation initiation by repressing the ability of the initiation factor eIF2 $\alpha$ to form the ternary complex with GTP and MettRNA $_{i}{ }^{\text {Met }}$. GCN1 does not interact with eIF2 $\alpha$ directly, but activates the eIF2 $\alpha$ kinase GCN2, which itself phosphorylates and inactivates eIF2 $\alpha$. Loss of function in gcn-1 should globally increase translation, and indeed Nukazuka et al. (2008) show that whole-animal levels of phospho-eIF $2 \alpha$ are decreased in their gcn-1 mutants. Somewhat unexpectedly, knockdown of the C. elegans GCN2 ortholog did not suppress plx-1, although it did reduce whole-animal phospho-eIF2 $\alpha$ levels; it is not known if the GCN1/GCN2 pathway defined from yeast is precisely conserved in C. elegans. In contrast, mutations in a second eIF2 $\alpha$ kinase, PEK-1/PERK, partially suppressed plx-1. gcn-1 pek-1 double mutants have very little phospho-eIF $2 \alpha$ and strongly (although not completely) suppress the ray 1 defects of $p l x-1$, suggesting that GCN-1 and PEK-1 act in parallel to phosphorylate eIF2 $\alpha$.

Reduction in $g \mathrm{cn}-1$ function results in bypass suppression of the smp/plx-1 ray 1 defects. Is this because SMP/ 
PLX-1 signals normally stimulate translation, or does increased translation act in parallel to compensate for lack of the SMP/PLX-1 signal? Whole-animal phospho-eIF $2 \alpha$ increases in plx-1 mutants, and decreases in animals conditionally overexpressing PLX-1 under the control of heat-shock promoters, consistent with PLX-1 signaling directly stimulating translation. To address whether semaphorins are specifically stimulating translation in the PLX-1-expressing ray precursors, Nukazuka et al. (2008) performed a tour de force of transgenic expression and biochemistry. By expressing tagged eIF $2 \alpha$ only in ray precursors, Nukazuka et al. (2008) show that loss or gain of PLX-1 function results in elevated or reduced phospho-eIF $2 \alpha$, and that the elevation in phospho-eIF $2 \alpha$ in ray precursors is dependent on the GCN-1 regulator and the PEK-1 kinase. Expression of a phosphomimetic (presumably inactive or dominant-negative) version of eIF2 $\alpha$ in ray precursors can phenocopy the ray 1 defect, whereas specific expression of a nonphosphorylatable and presumably activated eIF $2 \alpha$ suppressed the $p l x-1$ ray defect. These results convincingly demonstrate that the focus of regulated translation is autonomous to the ray cells.

Phospho-eIF $2 \alpha$ generally represses translation, but can also activate translation of specific messages, such as GCN4 in the yeast amino acid starvation response (Hinnebusch 2005). To address whether the effect of SMP/ PLX-1 signaling in ray 1 positioning requires increased or decreased translation, Nukazuka et al. (2008) performed RNAi of other factors required for translation initiation or elongation (eIF2, eIF4G, and eEF2), and found that these RNAi treatments phenocopied the $s m p / p l x-1$ ray 1 defect. Thus, PLX-1 signaling stimulates translation to promote normal ray 1 morphogenesis.

Suppression of plx-1 phenotypes by $g c n-1$ or by the gcn-1 pek-1 double mutant was not complete. These results seem most compatible with models in which PLX-1 signaling does not directly inhibit GCN-1 or PEK-1, but acts in parallel to reduce eIF2 $\alpha$ phosphorylation, perhaps by activating an eIF $2 \alpha$ phosphatase. Further, the low levels of phospho-eIF $2 \alpha$ in plx-1 gcn-1 pek-1 triple mutants did not result in complete restoration of a wild-type ray 1 , suggesting PLX-1 could also stimulate translation by a second mechanism in parallel to eIF $2 \alpha$. A candidate for such a parallel pathway would be the TOR/eIF4E pathway, shown to be activated by Sema3A in growth cone repulsion (Campbell and Holt 2001). Nevertheless, the finding that elevated translation bypasses the need for PLX-1, as well as previous evidence showing suppression of plx-1-null phenotypes (Dalpe et al. 2004), leads to the inevitable conclusion that localized SMP/PLX signaling is not itself essential for proper ray 1 positioning. Presumably pathways responsible for residual ray 1 positioning in plx-1-null mutants are able to position ray 1 properly in the suppressed double mutants.

\section{Semaphorins promote morphogenesis by stimulating ADF/cofilin translation}

To define the targets of SMP/PLX-1-stimulated translation in ray 1 morphogenesis, Nukazuka et al. (2008) tested known targets of semaphorin signaling in axon guidance. Semaphorin $3 \mathrm{~A}$ is known to collapse growth cones by depolymerizing actin via cofilin (Aizawa et al. 2001), and plexin C1 inhibits cell adhesion in murine dendritic cells via cofilin (Walzer et al. 2005). Recently, cofilin translation was shown to be induced rapidly by Sema3A in growth cones (Piper et al. 2006). Thus, in a variety of contexts, semaphorins can promote cofilinmediated actin depolymerization. In C. elegans, two $\mathrm{ADF} /$ cofilin isoforms, UNC-60A and UNC-60B, are encoded by the unc-60 gene (McKim et al. 1994). UNC-60A and UNC-60B are translated from alternatively spliced versions of the unc- 60 mRNA, yet are highly divergent in sequence; in fact, only the methionine is shared between the two ORFs. As UNC-60B is muscle-specific, Nukazuka et al. (2008) focused on UNC-60A, previously shown to be required in C. elegans cytokinesis (Ono et al. 2003). RNAi of unc- $60 \mathrm{~A}$ phenocopied the $p l x-1 / \mathrm{smp}$ ray 1 phenotype, consistent with a role for cofilin in ray 1 positioning. Furthermore, UNC-60A protein levels are decreased or increased in $p l x-1$ loss- and gain-of-function mutants, respectively, and transgenic expression of UNC-60A in ray precursors is sufficient to rescue plx-1 ray 1 phenotypes. These data convincingly show levels of UNC-60A as rate-limiting in ray 1 movement in the plx-1 mutants.

Somewhat unexpectedly, the unc- $60 \mathrm{~A}$ message is preferentially translated in Rn.p cells, relative to a more global activation of translation by SMP signaling. This preferential sensitivity is conferred by its $3^{\prime}$ untranslated region (UTR). Most of the previously studied cases of translational control involving the $3^{\prime}$ UTR are thought to involve interaction of $3^{\prime} \mathrm{UTR}$-binding repressors with components of the eIF4E (5' cap-binding) complex. Genes whose translation is repressed via the $3^{\prime}$ UTR have been studied extensively in C. elegans (e.g., tra-2, fem-3, and lin-14), although it must be admitted that in none of these cases have the relevant interacting translation factors yet been identified (Rhoads et al. 2006). Several possibilities may therefore be envisaged for how SMP/PLX-1 signaling might impinge on the unc-60 3'UTR. One novel possibility would be that the eIF $2 \alpha-$ GTP-tRNAi ternary complex is somehow sensitive to the $3^{\prime} \mathrm{UTR}$ repressor-binding factors, but that enhanced formation of the ternary complex as a result of eIF $2 \alpha$ dephosphorylation lifts it over a threshold that allows translation despite the inhibitory 3'UTR. Another possibility, although one that is harder to test directly, is that the role of the 3'UTR is more indirect, possibly in subcellular localization. In axon guidance, the 3'UTR of the RhoA message is required for its axonal targeting, and thus indirectly promotes sensitivity to Sema3A-stimulated translation (Wu et al. 2005). Current C. elegans in situ technology lacks the resolution to determine whether unc-60's 3'UTR might be affecting localization within the ray precursors. The unc-60 3'UTR contains a putative cytoplasmic polyadenylation element (CPE); it will be interesting to know whether this sequence is important in conferring sensitivity to translational stimulation.

$\mathrm{ADF} /$ cofilin is a potent actin depolymerization factor, 
and the results of Nukazuka et al. (2008) imply that actin depolymerization promotes the attraction of PLX-expressing ray cells to the SMP signal. UNC-60A has been shown to depolymerize actin, although interestingly it has a much lower actin-severing activity than UNC-60B (Yamashiro et al. 2005). However, cofilin also promotes growth cone collapse and repulsion (Piper et al. 2006), leading to the question of how actin depolymerization could promote both attractive and repulsive responses to semaphorin. Possibly, cycles of actin depolymerization and repolymerization are required for correct ray 1 cell movements within the epithelium. Such cycles are normally regulated by phosphorylation and dephosphorylation of cofilin; however, the relevant kinase and phosphatase (LIM-kinase and Slingshot) have not been identified in the C. elegans genome. The cellular basis of unc-60A function in this cell movement will be important to determine.

\section{Summary and future directions}

The work of Nukazuka et al. (2008) significantly extends our understanding of how semaphorins regulate epithelial morphogenetic movements. Translational control is emerging as an important mechanism in axon guidance (Lin and Holt 2007), in epithelial morphogenesis (Simoes et al. 2006), and in many other developmental processes (Sonenberg and Hinnebusch 2007). Strikingly, eIF2 $\alpha$ phosphorylation itself is also a control point in synaptic plasticity (Costa-Mattioli and Sonenberg 2006), suggesting that this might be a common pathway by which neurons regulate translation. However, other axon guidance cues such as ephrins do not appear to require local translation, and it remains unclear why regulated translation is used in some but not other pathways. The possible benefits of regulated translation in axon guidance include enhanced "local control" of axon choices, maintenance of axonal fate, and possible divergence in function between "old" and newly synthesized proteins (Lin and Holt 2007). In epithelial morphogenetic events such as the cell positioning considered here, it would seem that localized translation would be less of an advantage. However, another advantage may be in the potential for sustained responses to a transient signal. The exact timing of the semaphorin signal in ray positioning is unknown; Nukazuka et al. (2008) speculate that plexin may be activated continuously by semaphorins, in contrast to semaphorin signaling in growth cone collapse. However, if the SMP/PLX-1 signal is fleeting, then stimulation of translation might provide a more robust record of the interaction.

It will be interesting to see exactly how cofilin and the actin cytoskeleton function in the cell movements underlying ray positioning. It is striking that the same actin depolymerizing protein seems to be involved both in classic "repulsion" responses and in a process thought to be adhesive or attractive. However, this underscores our rather sketchy knowledge of the cellular basis of ray cell movements, either in the wild-type or in positioning mutants. Studies of the dynamics of ray precursor posi- tioning will shed important light on this type of morphogenetic cell movement.

\section{Acknowledgments}

I thank Yishi Jin for comments. Work in my laboratory is supported by the US Public Health Service (R01 GM54657).

\section{References}

Aizawa, H., Wakatsuki, S., Ishii, A., Moriyama, K., Sasaki, Y., Ohashi, K., Sekine-Aizawa, Y., Sehara-Fujisawa, A., Mizuno, K., Goshima, Y., et al. 2001. Phosphorylation of cofilin by LIM-kinase is necessary for semaphorin 3A-induced growth cone collapse. Nat. Neurosci. 4: 367-373.

Baird, S.E., Fitch, D.H., Kassem, I.A., and Emmons, S.W. 1991. Pattern formation in the nematode epidermis: Determination of the arrangement of peripheral sense organs in the $C$. elegans male tail. Development 113: 515-526.

Campbell, D.S. and Holt, C.E. 2001. Chemotropic responses of retinal growth cones mediated by rapid local protein synthesis and degradation. Neuron 32: 1013-1026.

Costa-Mattioli, M. and Sonenberg, N. 2006. Translational control of long-term synaptic plasticity and memory storage by eIF2 $\alpha$. Crit. Rev. Neurobiol. 18: 187-195.

Dalpe, G., Zhang, L.W., Zheng, H., and Culotti, J.G. 2004. Conversion of cell movement responses to Semaphorin-1 and Plexin-1 from attraction to repulsion by lowered levels of specific RAC GTPases in C. elegans. Development 131: 2073-2088.

Fujii, T., Nakao, F., Shibata, Y., Shioi, G., Kodama, E., Fujisawa, H., and Takagi, S. 2002. Caenorhabditis elegans PlexinA, PLX-1, interacts with transmembrane semaphorins and regulates epidermal morphogenesis. Development 129: 2053-2063.

Hinck, L. 2004. The versatile roles of 'axon guidance' cues in tissue morphogenesis. Dev. Cell 7: 783-793.

Hinnebusch, A.G. 2005. Translational regulation of GCN4 and the general amino acid control of yeast. Annu. Rev. Microbiol. 59: 407-450.

Ikegami, R., Zheng, H., Ong, S.H., and Culotti, J. 2004. Integration of semaphorin-2A/MAB-20, ephrin-4, and UNC-129 TGF- $\beta$ signaling pathways regulates sorting of distinct sensory rays in C. elegans. Dev. Cell 6: 383-395.

Lin, A.C. and Holt, C.E. 2007. Local translation and directional steering in axons. EMBO J. 26: 3729-3736.

Liu, Z., Fujii, T., Nukazuka, A., Kurokawa, R., Suzuki, M., Fujisawa, H., and Takagi, S. 2005. C. elegans PlexinA PLX-1 mediates a cell contact-dependent stop signal in vulval precursor cells. Dev. Biol. 282: 138-151.

McKim, K.S., Matheson, C., Marra, M.A., Wakarchuk, M.F., and Baillie, D.L. 1994. The Caenorhabditis elegans unc-60 gene encodes proteins homologous to a family of actin-binding proteins. Mol. Gen. Genet. 242: 346-357.

Nakao, F., Hudson, M.L., Suzuki, M., Peckler, Z., Kurokawa, R., Liu, Z., Gengyo-Ando, K., Nukazuka, A., Fujii, T., Suto, F., et al. 2007. The PLEXIN PLX-2 and the ephrin EFN-4 have distinct roles in MAB-20/Semaphorin 2A signaling in Caenorhabditis elegans morphogenesis. Genetics 176: 1591-1607.

Nukazuka, A., Fujisawa, H., Inada, T., Oda, Y., and Takagi, S. 2008. Semaphorin controls epidermal morphogenesis by stimulating mRNA translation via eIF2 $\alpha$ in Caenorhabditis elegans. Genes \& Dev. (this issue), doi: 10.1101/gad. 1644008 . 
Ono, K., Parast, M., Alberico, C., Benian, G.M., and Ono, S. 2003. Specific requirement for two ADF/cofilin isoforms in distinct actin-dependent processes in Caenorhabditis elegans. J. Cell Sci. 116: 2073-2085.

Piper, M., Anderson, R., Dwivedy, A., Weinl, C., van Horck, F. Leung, K.M., Cogill, E., and Holt, C. 2006. Signaling mechanisms underlying Slit2-induced collapse of Xenopus retinal growth cones. Neuron 49: 215-228.

Polleux, F., Morrow, T., and Ghosh, A. 2000. Semaphorin 3A is a chemoattractant for cortical apical dendrites. Nature 404: 567-573.

Rhoads, R.E., Dinkova, T.D., and Korneeva, N.L. 2006. Mechanism and regulation of translation in C. elegans. In WormBook (ed. The C. elegans Research Community), WormBook, doi: 10.1895/wormbook.1.63.1, http://www.wormbook.org.

Roy, P.J., Zheng, H., Warren, C.E., and Culotti, J.G. 2000. mab20 encodes Semaphorin-2a and is required to prevent ectopic cell contacts during epidermal morphogenesis in Caenorhabditis elegans. Development 127: 755-767.

Simoes, S., Denholm, B., Azevedo, D., Sotillos, S., Martin, P., Skaer, H., Hombria, J.C., and Jacinto, A. 2006. Compartmentalisation of Rho regulators directs cell invagination during tissue morphogenesis. Development 133: 4257-4267.

Sonenberg, N. and Hinnebusch, A.G. 2007. New modes of translational control in development, behavior, and disease. Mol. Cell 28: 721-729.

Tamagnone, L. and Comoglio, P.M. 2004. To move or not to move? Semaphorin signalling in cell migration. EMBO Rep. 5: 356-361.

Toyofuku, T., Zhang, H., Kumanogoh, A., Takegahara, N., Suto, F., Kamei, J., Aoki, K., Yabuki, M., Hori, M., Fujisawa, H., et al. 2004. Dual roles of Sema6D in cardiac morphogenesis through region-specific association of its receptor, PlexinA1, with off-track and vascular endothelial growth factor receptor type 2. Genes \& Dev. 18: 435-447.

Tran, T.S., Kolodkin, A.L., and Bharadwaj, R. 2007. Semaphorin regulation of cellular morphology. Annu. Rev. Cell Dev. Biol. 23: 263-292.

Walzer, T., Galibert, L., Comeau, M.R., and De Smedt, T. 2005. Plexin $\mathrm{C} 1$ engagement on mouse dendritic cells by viral semaphorin A39R induces actin cytoskeleton rearrangement and inhibits integrin-mediated adhesion and chemokine-induced migration. J. Immunol. 174: 51-59.

Wang, X., Zhang, W., Cheever, T., Schwarz, V., Opperman, K., Hutter, H., Koepp, D., and Chen, L. 2008. The C. elegans L1CAM homologue LAD-2 functions as a coreceptor in MAB-20/Sema2 mediated axon guidance. J. Cell Biol. 180: 233-246.

Wu, K.Y., Hengst, U., Cox, L.J., Macosko, E.Z., Jeromin, A., Urquhart, E.R., and Jaffrey, S.R. 2005. Local translation of RhoA regulates growth cone collapse. Nature 436: 1020-1024.

Yamashiro, S., Mohri, K., and Ono, S. 2005. The two Caenorhabditis elegans actin-depolymerizing factor/cofilin proteins differently enhance actin filament severing and depolymerization. Biochemistry 44: 14238-14247. 


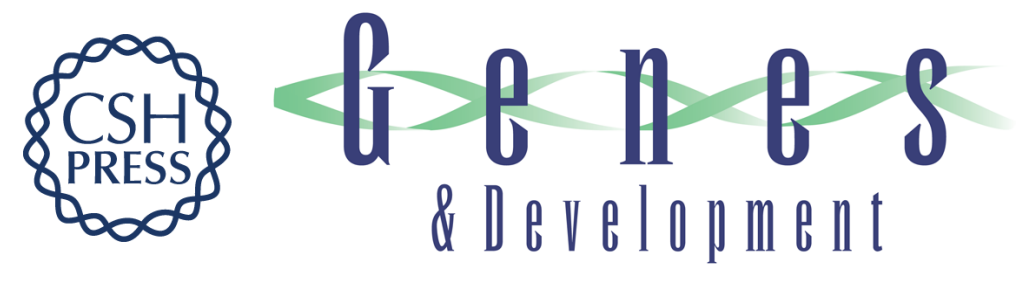

\section{Semaphorin signaling in morphogenesis: found in translation}

Andrew D. Chisholm

Genes Dev. 2008, 22:

Access the most recent version at doi:10.1101/gad.1669308
Related Content Semaphorin controls epidermal morphogenesis by stimulating mRNA translation via elF2 \pm in Caenorhabditis elegans
Akira Nukazuka, Hajime Fujisawa, Toshifumi Inada, et al.
Genes Dev. April , 2008 22: 1025-1036 Thinking Globally, Acting Locally
Annalisa M. VanHook
Sci. Signal. April , 2008 1: ec146
References This article cites 26 articles, 10 of which can be accessed free at:
http://genesdev.cshlp.org/content/22/8/955.full.html\#ref-list-1
Articles cited in:
http://genesdev.cshlp.org/content/22/8/955.full.htmI\#related-urls

\section{License}
Email Alerting
Receive free email alerts when new articles cite this article - sign up in the box at the top Service right corner of the article or click here.

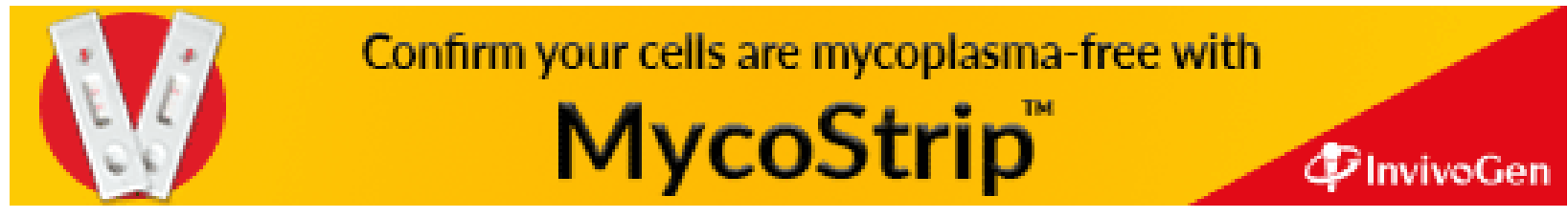

